FORMATION Formation emploi

Revue française de sciences sociales

131 | Juillet-Septembre 2015

Le Bac Pro a 30 ans

\title{
Les inégalités scolaires par l'alternance ? Deux déclinaisons de la littératie dans l'enseignement agricole
}

Educational inequality in sandwich courses. Two variations of literacy in agricultural education

Schulische Ungleichheiten durch duales System ? Zwei Deklinationen von Lernund Schreibfähigkeit im landwirtschaftlichen Bildungswesen ¿Las desigualdades escolares por la alternancia? Dos declinaciones de la alfabetización en la enseñanza agrícola

\section{Laure Minassian}

\section{OpenEdition}

Journals

Édition électronique

URL : http://journals.openedition.org/formationemploi/4465

DOI : 10.4000/formationemploi.4465

ISSN : 2107-0946

Éditeur

La Documentation française

Édition imprimée

Date de publication : 15 octobre 2015

Pagination : 61-77

ISSN : 0759-6340

Référence électronique

Laure Minassian, «Les inégalités scolaires par l'alternance ? Deux déclinaisons de la littératie dans l'enseignement agricole », Formation emploi [En ligne], 131 | Juillet-Septembre 2015, mis en ligne le 15 octobre 2017, consulté le 30 octobre 2020. URL : http://journals.openedition.org/formationemploi/ 4465 ; DOl : https://doi.org/10.4000/formationemploi.4465 


\title{
Les inégalités scolaires par l'alternance? Deux déclinaisons de la littératie dans l'enseignement agricole
}

\begin{abstract}
Laure Minassian
Docteur en science de l'éducation, elle fait partie du Centre Interdisciplinaire de Recherche Culture Éducation Formation Travail Education et Scolarisation (CIRCEFT-ESCol) de Paris VIII. Sa thèse porte sur les inégalités sociales et scolaires dans l'enseignement agricole.
\end{abstract}

Résumé

Les inégalités scolaires par l'alternance? Deux déclinaisons de la littératie dans l'enseignement agricole

Les formations par alternance sont souvent préconisées pour aider des élèves en échec dans les dispositifs scolaires plus ordinaires. Mais les effets de ces dispositifs sont-ils ceux attendus ? Deux systèmes d'alternance contrastés dans l'enseignement agricole sont comparés. L'analyse porte sur quatre-vingt-dix dossiers rédigés par des élèves, à différents moments de leur parcours de formation. Le cadre d'analyse s'inspire des new litteraties studies, pour étudier quelques relations entre les usages de l'écrit dominants dans ces deux systèmes et à quels types d'inégalités ces usages peuvent être associés.

Mots-clés : enseignement agriculture, formation en alternance, baccalauréat professionnel, enseignement privé, compétence, lycée professionnel.

Abstract

Educational inequality in sandwich courses? Two variations of literacies in agricultural education

The sandwich courses are often recommended to help short comers in more mainstream schools. But the effects of these devices are-there those expected? Two types of sandwich courses in agricultural education are compared. The analysis covers ninety records written by students at different points in their training. The framework, inspired by the current of new literacies studies, is used to investigate some relations between the uses of writing in these two institutions, types of pupils' types and educational inequality.

Key words: education by the ministry of agriculture, sandwich training, vocational baccalaureate, private education, skill, vocational upper secondary school.

Journal of Economic Literature: I 21

Traduction : Auteur. 
L'enseignement agricole s'est, en grande partie, constitué à partir d'établissements et d'expérimentations pédagogiques privés. Si la plupart de ces établissements se sont institutionnalisés par une intégration officielle dans le système éducatif à la fin des années 1950 (contractualisation dans le cadre de la loi Debré de 1959), d'autres ont échappé à cette normalisation et ont continué à cultiver leur singularité jusqu'au milieu des années 1980. C'est le cas d'un groupe d'écoles associatives : les Maisons Familiales Rurales (MFR), créées en 1937 (Loi Rocard, 1984 ; Lelorrain, 1999).

La spécificité des MFR, depuis ce temps, a perduré sous des formes quelque peu différentes, en particulier dans leurs principes pédagogiques, subsumés sous l'idée que l'école ne peut être séparée de la vie et ainsi du travail (Chartier, 1986). Les politiques publiques ,en recherche de solutions pédagogiques pour favoriser le retour de jeunes sans qualification ou peu qualifiés vers l'école, s'y sont intéressé. L'enseignement agricole vise ainsi à servir des objectifs d'égalité, d'efficacité et de remédiation en diversifiant son offre qui va des filières métiers du bois à celles des services à la personne, en passant par l'enseignement des productions agricoles. On se demandera jusqu'à quel point ces objectifs sont atteints, compte tenu, en particulier, de la spécificité relative du public accueilli.

Notre enquête ethnographique dans deux classes, l'une de MFR, la seconde d'un lycée privé, apporte une réponse mitigée. Les deux classes retenues sont de même niveau de formation, les élèves y préparent le baccalauréat professionnel «Conduite et Gestion d'une Exploitation Agricole " (CGEA), c'est-à-dire le diplôme le plus emblématique de l'enseignement agricole.

Conçu spécifiquement pour les futurs exploitants agricoles, ce diplôme masque des disparités d'autant plus vives que loin d'être majoritaires dans l'enseignement agricole, les enfants d'agriculteurs côtoient des enfants d'employés et d'ouvriers devenus les plus nombreux.

Cet afflux d'élèves se double d'une division sociale d'orientation : dans les classes observées, les enfants d'agriculteurs sont majoritaires dans la classe du lycée, tandis que les élèves de la MFR observée sont surtout extérieurs à ce milieu, sans que l'on puisse mesurer avec précision la part de stratégie des familles et la part de politique de recrutement des établissements (Minassian, 2015).

La séparation sociale des élèves semble s'accompagner d'une division sociale du travail cognitif. C'est du moins ce que semble suggérer une analyse basée sur la notion de littératie, définie comme les habitudes cognitives et langagières construites dans les pratiques scripturales socialement situées des élèves. L'écrit est en effet un lieu privilégié de mise à distance des pratiques professionnelles et des savoirs issus du stage.

Plus précisément, comme le montre le courant des new litteraties studies qui s'est intéressé aux variations des pratiques de l'écrit et à leurs effets cognitifs, les modes de faire avec l'écrit ne surgissent pas ex nihilo, mais sont influencés par l'environnement socioculturel 
(Heath, 1986 ; Street, 1984 ; 2003). Dans cette perspective, l'institution d'alternance, d'une manière ou d'une autre, participe à l'évolution des écrits.

Les relations entre pédagogies de l'alternance, littératies et inégalités seront présentées en quatre temps. Après avoir souligné combien des intitulés de diplômes identiques peuvent masquer une diversité d'établissements et de dispositifs, la mobilisation d'un cadre théorique autour des questions de l'écrit servira à étudier, dans le détail, des pratiques d'élèves in situ. Dans un troisième temps, l'étude des écrits des élèves, à partir de quatre-vingt-dix dossiers qu'ils ont rédigés, permettra de dégager les traits saillants d'une hétérogénéité de dispositions à l'œuvre d'un groupe à l'autre. La coexistence d'une pluralité de littératies scolaires avec différents dispositifs pédagogiques de l'alternance sera enfin discutée.

\section{Sous des diplômes identiques, une diversité d'établissements}

Les différents établissements d'alternance préparent à des diplômes dont les intitulés sont généralement unifiés, du CAP (certificat d'aptitude professionnelle) au bac pro et au bac technologique. Pourtant, la mise en lumière de l'alternance comme "notion polymorphe " (Agulhon, 2000 ; reprise par Merhan, Ronveaux, Vanhulle, 2007) a mis en exergue une pluralité d'établissements et de pratiques.

\section{Encadré 1 : Un lycée à la campagne et une Maison Familiale Rurale « urbaine »}

Une manière de distinguer les deux établissements retenus consiste à les rapporter à l'opposition urbain/rural. La MFR est construite aux abords d'une ville de plus de 15000 habitants dans l'agglomération nantaise, tandis que le lycée se situe dans une commune qui compte environ 3500 habitants, en pleine campagne. La situation géographique actuelle de la MFR est stratégique : vingt ans auparavant, cet établissement se situait dans une autre commune de 400 habitants. Sa nouvelle localisation, en périphérie d'une ville, près d'une quatre voies et d'une zone commerciale, permet de toucher un grand nombre d'élèves potentiels, d'origine urbaine ou rurale. Un ensemble de services d'aides à la mobilité sont proposés : outre un internat (obligatoire dans toute MFR), l'école a mis en place un service de minibus pour accueillir les élèves à la gare le dimanche soir et les raccompagner le vendredi soir.

Par contraste, le lycée agricole est situé dans une commune rurale privilégiée par une tradition aristocratique et de notables favorables à l'implantation d'une école agricole tenue par la congrégation des Frères de I'Instruction Chrétienne de Ploërmel, dès le milieu du XVIIlème siècle. À cet effet, de nombreuses terres agricoles ont été offertes à cette communauté religieuse. Le lycée agricole est situé au cœur du village. Dans cette commune, outre ce lycée, les Frères ont en charge l'école maternelle, l'école primaire et le collège (le seul de tout le canton dans lequel il n'existe pas de collège public). Toutes ces écoles sont situées sur le même côté de la rue, dans l'ordre suivant à partir de l'église : la maternelle, l'école primaire, le collège et le lycée agricole. Cette implantation spatiale place ce lycée dans une continuité scolaire naturalisée. 
Cette pluralité caractérise les écoles dans lesquelles nous avons enquêté en Loire-Atlantique. Elles se distinguent au moins par le temps d'alternance : les stages occupent jusqu'à $50 \%$ du temps pédagogique en MFR (L. 813-9), contre $25 \%$ au lycée ${ }^{1}$. On aurait tort de réduire cette différence à un enjeu seulement temporel, car elle contribue à la répartition des élèves : les élèves les plus en difficultés scolaires s'inscrivent dans des "écoles de demitemps " (Charlot et Figeat, 1985) quand les autres privilégient le temps scolaire. De plus, l'organisation temporelle se double d'une organisation spatiale selon que prévaut l'ouverture ou le cloisonnement des lieux. Et même si les deux établissements observés sont proches géographiquement, ils possèdent des conditions matérielles d'existence éloignées.

Sur le plan architectural, les bâtiments du lycée agricole sont construits sur le modèle des anciens lycées. L'entrée de l'établissement est imposante, la cour se situe dans l'enceinte de l'établissement, les fenêtres des classes donnent le plus souvent sur la cour. La MFR est en revanche une école plus récente, les locaux sont davantage disséminés sur un espace vert non délimité.

Sur le plan des usages internes des locaux, on retrouve d'autres oppositions. Au lycée, les élèves ont des lieux dédiés (la cour, le foyer en sous-sol). Ils ne peuvent accéder à la salle des professeurs, le couloir y menant leur étant strictement interdit, comme pour marquer les relations hiérarchiques entre ces différentes catégories d'usagers de l'école. Par contraste, les élèves de MFR ont facilement accès aux bureaux des enseignants, leur foyer est au cœur de l'école et constitue un lieu de passage obligé pour accéder à certaines salles de cours. Autrement dit, les lieux destinés aux apprentissages scolaires et ceux destinés aux loisirs sont entremêlés. Les espaces affectés aux élèves et aux enseignants (internat, foyer, classes, bureaux) sont peu séparés, sur le principe d'une maison de famille.

Cette organisation est cohérente avec les types de relations élèves-enseignants. En MFR, les enseignants sont tenus à une certaine proximité avec les élèves : dans leurs heures de service, ils doivent partager des repas à la cantine avec ces derniers, mais aussi des sorties, des temps de veillées, d'étude, alors qu'au lycée, pourtant pourvu d'un internat, ces temps hors de la classe n'existent pas et la séparation d'avec les élèves est plus marquée.

Cette opposition n'est pas sans rappeler les travaux de Panofsky (1967) qui montraient une affinité entre architecture et dispositions culturelles ${ }^{2}$. D'autres travaux tels que ceux de Bernstein ont aussi souligné une proximité entre les formes spatiales au travers des notions "écoles ouvertes" et "écoles fermées", avec respectivement des pédagogies progressistes et académiques à l'œuvre (Bersntein ; 1975). L'auteur dénonçait ces situations où la pédagogie se fait plus conciliante dès lors que les élèves sont en difficulté, plus exigeante quand ils réussissent. L'implantation des bâtiments, les usages des locaux consti-

1. Dans les deux formations observées, les élèves effectuent leur stage sous le régime du statut scolaire.

2. L'école, construite sur un modèle vertical, renvoie aux traits architecturaux des cathédrales, c'est-à-dire à la séparation explicite des différentes parties, «à la clarté des hiérarchies» (Déotte, 2010). 
tuent déjà des indicateurs de la manière dont l'institution normalise son public tout en se conciliant les individus.

Par conséquent, derrière l'accès aux diplômes, aux mêmes spécialités d'enseignement, aux mêmes classes, sous l'identique donc, se joue un grand nombre de différences de traitement qui, à la longue, rétroagissent sur le recrutement.

Ainsi, dans les classes suivies, avant même l'entrée en formation, les trajectoires des élèves sont contrastées. Dans la classe de MFR, les parcours des élèves sont non linéaires, ponctués de redoublements et d'orientations précoces (parfois dès la cinquième), c'est-à-dire très différents de ceux plus ordinaires des élèves du lycée. En effet, ces derniers ont peu redoublé, ont suivi une troisième générale et obtenu le diplôme national du brevet (sauf un).

Ces trajectoires scolaires sont aussi liées à l'origine sociale car si les enfants d'employés et d'ouvriers sont aujourd'hui majoritaires dans l'enseignement agricole (DGER, 2013) en raison du déclin de la paysannerie, ils sont massivement représentés en MFR et peu présents au lycée : seuls quatre élèves sur quinze dans la classe de MFR étaient fils d'agriculteurs, alors qu’ils l'étaient tous dans celle du lycée.

\section{Pratiques sociales de l'écrit : 2 une occasion pour mettre à distance l'expérience}

La signification sociale de ce que construisent les élèves peut se révéler grâce à l'analyse de leurs pratiques écrites. À la suite des perspectives théoriques ouvertes par les new litteraties studies, l'écrit n'est pas considéré ici comme un simple moyen technique de communication ; il est en effet envisagé comme une activité humaine englobant un ensemble de pratiques qui distinguent les individus dans les manières d'en faire usage, de mobiliser certains registres plutôt que d'autres, dans les façons de nouer des relations entre des objets de savoir et donc de se les approprier. L'écrit se caractérise par son caractère différé, par le recours nécessaire à l'explicitation, tandis que les interactions constitutives de situations orales autorisent des élisions, du para-verbal, du méta-discours, des retours en arrière à la demande, des intonations.

En outre, comme le montre un ensemble de travaux anthropologiques, l'écriture implique un changement de statut des énoncés, en apparence infime, par le passage de l'oreille à l'œil (Goody, 2007). Elle autorise un premier détachement du sujet et ouvre potentiellement un champ des possibles de par sa matérialité. L'observation à distance facilite un processus de manipulation des énoncés pour les reprendre et les transformer et ainsi les objectiver (Lahire, 1993).

L'écrit est donc une occasion en puissance, par le temps affranchi des contraintes matérielles qu'il convoque, de mettre à distance les objets du travail puisqu'ils sont ainsi dégagés des 
"urgences de la nécessité " (Bourdieu, 1996). Les éléments langagiers sur lesquels reposent nos analyses visent à rendre compte de la manière dont les élèves mettent en relation les objets qu'ils mobilisent, des liens qu'ils construisent entre ces objets comme indices de création de sens. Les indices langagiers relevés et leur cumulation permettent de décrire des manières de faire avec l'écrit, de mettre au jour quelques éléments d'une littératie à l'œuvre en tant qu'activité socialement et culturellement située (Street, 1993).

\section{Encadré 2. Littératies et cognition}

L'anthropologue Goody définit l'écrit comme « technologie de l'intellect» (Goody ; 2007). Cet outil cognitif autorise, par exemple, à substituer la succession mnémotechnique des événements de la journée, de l'année ou encore des saisons, par un autre mode de fonctionnement. Dès lors que la succession peut être couchée par écrit, il n'est plus pertinent de l'apprendre puisqu'elle peut être vue. Cette mise à distance où l'œil peut saisir, dans l'espace restreint constitué par la page, le temps, mais aussi l'espace grâce à la cartographie, ou encore un raisonnement, une description, est une occasion nouvelle d'agencer autrement les éléments, de les reconstruire, de catégoriser le monde.

D'autres auteurs ont souligné que l'apprentissage du lire-écrire n'accroît pas en soi la réflexivité. Les formes d'abstraction se manifestent plutôt dans la manière de s'en saisir, dans ce qui en est fait (Schribner, Cole ; 1981). Dans la même perspective, Budach et Patrick (2012), tout comme Barton et Hamilton (2010), montrent que les pratiques écrites ne peuvent être considérées pour elles-mêmes, au moins parce qu'elles sont façonnées par l'environnement immédiat dans lequel elles sont inscrites ainsi que par les mouvements et trajectoires des individus. Autrement dit, l'écrit est, comme toute activité, une pratique sociale. Enfin, pour Street $(1984 ; 1995)$ et Heath (1986), les littératies résultent des processus d'interactions entre des ressources symboliques et l'éventail de dispositions des sujets (Bautier, 2009).

Le concept de littératie est ici repris pour deux raisons. En premier lieu, il permet de comprendre en quoi et comment les élèves se saisissent des objets quotidiens qu'ils fréquentent sur le lieu du stage, par exemple, et ce qu'ils font avec ces objets dans l'écrit, sachant que le passage d'une communication efficace en situation de travail à celui d'une reprise réflexive dans l'écrit ne va pas de soi (Boutet, 2008). En second lieu, les manières de faire avec l'écrit s'inscrivent dans une multiplicité d'éléments qui composent l'environnement des sujets, même si ceux-ci les réordonnent subjectivement. Ce dernier élément justifie l'hypothèse selon laquelle l'institution de formation constitue un élément de l'environnement des scripteurs et, à ce titre, joue un rôle dans l'évolution des pratiques scripturales.

\section{Des productions écrites comme indices de 3 raisonnements à l'œuvre... et de leur inachèvement}

Pendant deux années, deux groupes inscrits en bac pro CGEA (Conduite et Gestion d'une Exploitation Agricole) ont été suivis. Ce baccalauréat agricole, qui a remplacé le BTA (Brevet de Technicien Agricole), a été créé afin d'assurer un niveau de connaissances techniques et théoriques minimum préalablement à l'installation d'une exploitation agricole. 
Ce diplôme de niveau IV constitue, à ce titre, ce que l'on nomme, dans la culture commune, "le sésame ", et permet d'obtenir des aides financières à l'installation.

Le module professionnel étudié (Production Animale) occupe une position d'interface dans le curriculum, à deux points de vue. Tout d'abord, il est le plus en relation avec le stage, permettant idéalement la mise en œuvre d'une pédagogie ouverte sur le monde du travail dont se réclament les MFR. Ensuite, il possède le plus fort coefficient pour l'obtention du baccalauréat, requérant ainsi, de la part des élèves, un investissement dans l'écrit à composante professionnelle : ils doivent rédiger des dossiers thématiques sur les pratiques d'élevage. Le corpus est constitué de tels dossiers qui tiennent aussi lieu de copies d'examen en "Contrôle en Cours de Formation " (CCF). Pendant deux ans, tous les dossiers des élèves ont été recueillis (300 dossiers, soit plus de 3000 feuilles).

Dans cette masse d'information, nous avons choisi de nous intéresser à trois moments charnières de la formation (le début, la fin et à mi-parcours), soit 90 dossiers. Dans le cadre restreint de cet article ne seront mobilisés que les dossiers les plus démonstratifs parmi les 300 recueillis.

Outre le fait qu'il existe un flou dans le référentiel de formation, en concordance avec une tendance curriculaire générale (Forquin, 2008 ; Mangez, 2008), la notation officiellement différente d'une classe à l'autre accroît ce flou. En effet, les rédacteurs du référentiel laissaient à l'enseignant le choix de la part de la notation de l'écrit : la note de l'écrit pouvait occuper entre $30 \%$ et $70 \%$ de la note finale d'examen. Quelle que soit l'importance accordée à l'écrit ${ }^{3}$, les caractéristiques des littératies produites dans un contexte d'alternance peuvent être objectivées, comme pour toute activité scripturale, et révéler une double dimension, socio-cognitive, d'une part, et socio-culturelle, d'autre part.

Sur le plan cognitif, le principe selon lequel chaque discours engendre ses propres catégories d'analyse justifie la démarche à dominante inductive ici mobilisée (Normand, 2014). Les schèmes organisateurs retenus afin d'étudier la structure et le contenu des discours, les univers auxquels ces contenus renvoient ont été choisis à partir du corpus lui-même. Certains éléments inter-reliés ressortent de façon significative :

- La présence d'enchaînement des énoncés pour construire un texte articulé autour d'un thème, ou au contraire l'absence d'enchaînements propres aux énoncés saccadés ;

- La reprise de l'expérience, des normes, par un questionnement, des savoirs explicites, par opposition à la convocation de routines du travail agricole comme justification in se d'une conduite à tenir, de vécus sur le lieu de stage, des normes comme allant de soi ;

- Les types de nominalisation des objets comme indices d'une mise à distance ou non de l'objet ;

3. La part accordée à l'écrit dans la note finale était de $70 \%$ dans le lycée ; nous n'avons pas pu savoir quelle était cette part dans la MFR. 
- Le mode énonciatif adopté par les élèves à partir d'inflexions, de tons dans l'écrit, comme dénotant un effacement énonciatif au profit de la construction d'un texte plus générique et réflexif, ou, au contraire de mise en avant de soi ou du maitre de stage.

Le cumul de ces indicateurs n'est pas sans lien avec l'hypothèse selon laquelle, à travers l'enchaînement des énoncés, peuvent être reliées les phases de l'alternance, et en particulier l'expérience du travail agricole et le savoir savant. La spécificité des énoncés, leur caractère disparate ou décousu, sur le modèle des conversations ordinaires ou les conversations au travail étudiées par Boutet (2008), la place qu'ils occupent dans la chaîne de l'écrit, sont autant d'indicateurs de la position graduée de l'élève, quelque part à mi-chemin entre le terrain et le savoir scolaire.

\section{Encadré 3. Les limites de l'enquête}

L'enquête proposée est une étude de cas conduite sur deux groupes restreints d'élèves socialement distincts et scolarisés dans des établissements contrastés. Ces différences constituent un avantage au sens où elles permettent de montrer ce que fait l'institution scolaire une fois qu'elle a achevé le classement des élèves dans des dispositifs scolaires hiérarchisés. En revanche, ce choix d'enquête ne permet pas de rendre compte d'éléments plus génériques relativement à l'enseignement agricole et à l'alternance. Parce que nous avons choisi d'observer des dispositifs très différents dans l'éventail des dispositifs disponibles, les résultats d'enquête obligent à une certaine prudence.

\section{Transiger avec les écrits des élèves : un facteur d'émancipation?}

La manière dont les élèves se saisissent de l'écrit permet d'éclairer des rapports aux pratiques du stage et de l'école socialement situés. Un premier plan d'analyse est constitué à partir des manières de rédiger, donnant lieu à un texte cohérent, à un raisonnement ou au contraire à des réponses hâtives comme pour s'acquitter du travail demandé. Le tableau 1 présente les fréquences de ces deux types d'énoncés dans une perspective longitudinale. Un second plan d'analyse est formé par l'étude des effets que produisent les expressions très brèves. Très souvent, celles-ci font obstacle au recours à un langage décontextualisé (Olson, 1977), alors que l'usage d'un langage explicite hors contexte contribue à une réflexivité par ailleurs de plus en plus exigée dans les référentiels de formations professionnelles. Enfin, sur le plan des univers cognitifs mobilisés dans l'écrit, une expertise puisant dans la science agricole appliquée n'est pas de même niveau que l'expérience vécue empiriquement et rapportée dans les mêmes termes. 


\subsection{Relier les énoncés pour relier les situations de formation hors et dans la classe}

L'enchaînement, ou le non-enchaînement, des énoncés constitue un indicateur de la capacité à relier les éléments pour produire un discours logiquement structuré. Alors que certains élèves produisent des énoncés segmentés donnant lieu à un discours haché, d'autres tentent de les faire circuler ensemble pour construire un texte. Les deux exemples ci-dessous permettent d'illustrer cette distinction. Dans l'exemple 1, les énoncés sont posés successivement sans relation entre eux. Portant sur les objectifs d'un éleveur, ils signifient que l'élève n'associe pas les différents objectifs à atteindre, ou du moins que son écrit ne manifeste pas ce type d'association. Dans l'exemple 2, un autre élève met au contraire en relation des éléments de contexte d'une exploitation avec des pratiques d'élevage pour construire une problématique :

\section{Exemple 1 : énoncés non-enchaînés}

"Les objectifs de l'éleveur.

- L'exploitant utilise et valorise au printemps ses fourrages et le pâturage.

- L'utilisation des fourrages stockés se fait par quantité et non par quantité.

- Les concentrés interviennent en plus des fourrages pour l'équilibre azotelénergie et la production laitière. " (Bastien)

\section{Exemple 2 : énoncés enchaînés}

"Sur cette exploitation la SAU (Surface Agricole Utile ${ }^{5}$ ) représente 125 ha (hectares) principalement constitués d'herbe car la surface en herbe représente $60 \%$ de la SAU et $72 \%$ de la SFP (Surface fourragère Principale). Mais comparativement à cela la surface pour le pâturage est de 25 ha pour le cheptel laitier soit $24 \%$ de la SFP. Pourquoi un tel choix?" (Jordenn)

L'ensemble des énoncés enchaînés et non-enchaînés a fait l'objet d'une quantification ( $c f$. tableau 1). La comparaison porte sur deux séries (1 241 énoncés) et permet de rendre compte d'évolutions entre le début et la fin de la formation.

4. Lélève utilise deux fois le terme " quantité ", l'un des deux termes est possiblement à remplacer par le terme " qualité ".

5. Le discours de l'élève relevant d'un registre technique, nous avons mis entre parenthèses la signification des acronymes comme aide à la lecture. 
Tableau 1. Indicateurs de construction des arguments : énoncés avec ou sans enchaînement (catégorisation à partir de 1241 énoncés)

\begin{tabular}{|c|c|c|c|c|c|c|}
\hline Institutions & & MFR & & & Lycée & \\
\hline $\begin{array}{l}\text { Catégorisation des énoncés } \\
\text { dans les dossiers des élèves }\end{array}$ & $\begin{array}{c}\text { Énoncés } \\
\text { non- } \\
\text { enchaînés }\end{array}$ & $\begin{array}{l}\text { Énoncés } \\
\text { enchaînés }\end{array}$ & Total & $\begin{array}{c}\text { Énoncés } \\
\text { non- } \\
\text { enchaînés }\end{array}$ & $\begin{array}{l}\text { Énoncés } \\
\text { enchaînés }\end{array}$ & Total \\
\hline $\begin{array}{l}\text { Dossier en début de classe } \\
\text { de } 1^{\text {ère }} \text { pro }\end{array}$ & $290(68 \%)$ & $\begin{array}{c}135 \\
(32 \%)\end{array}$ & $\begin{array}{c}425 \\
(100 \%)\end{array}$ & $\begin{array}{c}199 \\
(59,0 \%)\end{array}$ & $\begin{array}{c}138 \\
(41 \%)\end{array}$ & $\begin{array}{c}337 \\
(100 \%)\end{array}$ \\
\hline $\begin{array}{l}\text { Dossier en fin de terminale } \\
\text { pro }\end{array}$ & $\begin{array}{c}217 \\
(82 \%)\end{array}$ & $\begin{array}{c}47 \\
(18 \%)\end{array}$ & $\begin{array}{c}264 \\
(100 \%)\end{array}$ & $\begin{array}{c}83 \\
(39 \%)\end{array}$ & $\begin{array}{c}132 \\
(61 \%)\end{array}$ & $\begin{array}{c}215 \\
(100 \%)\end{array}$ \\
\hline
\end{tabular}

Source : Enquête auteure. MFR : maison familiale rurale.

Les deux groupes d'élèves présentent des traits communs et des différences. Sur le plan de la longueur de l'écrit, il apparaît que les élèves de MFR composent des dossiers plus longs que leurs homologues du lycée, au début comme à la fin de la formation, alors qu'ils ont connu des trajectoires scolaires plus atypiques.

Deux d'hypothèses peuvent expliquer cet écart. Tout d'abord, certains élèves dits en difficultés scolaires peuvent avoir des niveaux de performance proches des élèves scolarisés dans des classes ordinaires (Zaffran, 2010, Goigoux, 2000). On peut aussi supposer que les élèves de MFR s'investissent scolairement en produisant des écrits plus longs et expriment ainsi leur envie de réussir là où ils ont précédemment échoué. En ce sens, ils ne sont pas, au début de la formation, en résistance par rapport à l'école, mais développent un rapport plus complexe qu'on ne peut rapporter à une échelle graduée sur le modèle proximité-distance-résistance.

Cette double hypothèse semble confirmée par le fait que le taux d'énoncés enchaînés entre les groupes est relativement proche : $32 \%$ en MFR, pour $41 \%$ au lycée. Mais la prudence s'impose, puisque cet écart est aussi généré par les types de consignes auxquels les élèves sont confrontés. Etant donné que ces consignes ne sont pas tout à fait équivalentes d'une classe à l'autre, elles peuvent être un facteur explicatif des différences constatées. En outre, on peut supposer qu'en dépit d'inégalités de parcours, les performances globalement comparables peuvent être imputées à l'effet correcteur de la scolarisation, en l'absence d'effet propre de l'alternance à ce stade.

En revanche, les énoncés enchaînés des élèves de MFR diminuent significativement de près de la moitié, alors qu'ils augmentent pour les élèves du lycée. La comparaison entre les énoncés enchaînés et non-enchaînés fait apparaître des variations inversement proportionnelles entre les groupes.

Ces éléments, comparés entre début et fin de formation, amènent à interroger les pédagogies de l'alternance et leur adaptation aux parcours d'orientation des élèves. Car alors qu'en MFR, la trajectoire scolaire des élèves est balisée par un ensemble de difficultés, les perfor- 
mances des deux groupes ne sont pas si éloignées au début de la formation. Cependant, les écarts deviennent très importants au fur et à mesure des deux années. Le niveau de classe et la spécialité observés étant communs aux deux dispositifs, en $1^{\mathrm{e}}$ et terminale bac pro "Conduite et Gestion de l'Exploitation Agricole », les écarts constatés seraient alors imputables au déroulement de l'alternance et aux pratiques pédagogiques associant temps scolaire et temps de stage.

\subsection{Amorcer une réflexivité à l'égard de l'expérience de stage}

Des différences qualitatives apparaissent ainsi en de nombreux endroits. Au début de la formation, les élèves de MFR tentent de mettre en avant les intérêts et les limites d'une pratique d'élevage, par exemple, c'est-à-dire d'en construire des indices de sens (les textes des élèves sont reproduits fautes comprises, ainsi qu'avec l'ensemble des éléments sémiotiques ; flèches, symboles etc.) :

"Les objectifs des l'éleveurs

Tout d'abord, le principal objectif est de produire le quota ainsi que les $\longrightarrow$ augmentations que la laiterie donne.

Pour ce qui est de la productivité de l'élevage, l'exploitant recherche :

- au niveau génétique : mamelle, morphologie, fertilité, lait et taux

- au niveau production : recherche un niveau de production à 8500-9500/VL/an

- favoriser le pâturage

- maintenir le maïs toute l'année

- garder suffisamment de blé pour l'autoconsommation " (Quentyn).

À la fin de la formation, les élèves de MFR ne rédigent plus que des réponses très brèves, le plus souvent sous forme de tableau :

\begin{tabular}{|l|l|}
\hline Atouts & Contraintes \\
$\begin{array}{l}\text { Bâtiment clair } \\
\text { Lumineux }\end{array}$ & Vents sud (froids) \\
\hline
\end{tabular}

(Valentyn)

\begin{tabular}{|l|l|}
\hline Méthodes & $\begin{array}{l}\text { IA }\left(^{*}\right) \text { (avec achat de paillettes extérieures et sexées), réalisé } \\
\text { par l'inséminateur } \\
\text { Transplantation sur génisses (depuis } 15 \text { ans environ) }\end{array}$ \\
\hline Repérage et enregistrement & $\begin{array}{l}\text { Poket et logiciel EDIPA } \\
\text { Repérage par observation et suivi avec le Poket }\end{array}$ \\
\hline
\end{tabular}

(Jules)

$(*)$ : IA : insémination artificielle. 
Le dossier à mi-parcours de la formation semble illustrer un moment charnière de ces deux modes d'énonciation. Dans ces dossiers, les élèves rabattent leurs écrits sur la narration d'objets observables, rédigent des descriptifs que pourrait réaliser un visiteur quelconque sur une exploitation. Ils rapportent ce qu'ils voient empiriquement, parfois même les propos du maître de stage :

"Tout les soirs l'agriculteurs vient voir ses vaches (vers 22h) pour repérer les vaches en chaleur. Si une vache n'est pas en chaleur au bout de 50 jours, elle est contrôlée par l'inséminateur et le vétérinaire" (Ewann).

"Repérage des chaleurs: ils sont tous repérés grâce à la vue, et si les vaches sont en chaleur, on regarde sur le bulletin du contrôleur laitier si elles sont bonnes à inséminer "(Julien).

" (...) l'éleveur à sa maison à côté, se qui permet de surveiller aisément, les mises bas" (Jérémy).

"Les vêlages ont le plus souvent lieu au pré, avec une observation régulière. Les vêlages se font sans aide, lorsque tout se présente bien. L'éleveur préfere laisser faire la nature " (Jérôme).

"La surveillance des chaleurs est également réalisée le matin, le midi et le soir, en restant avec les animaux environ 15 minutes" (Valentyn).

Par contraste, les élèves du lycée soulignent des écarts entre des éléments constatés et en construisent une problématique. Cette tendance est beaucoup moins fréquente pour les élèves de MFR (excepté pour deux d'entre eux sur quinze) :

"Tout d'abord l'intervalle vêlage lère IA (première Insémination Artificielle) paraît réparti à près de $50 \%$ sur les données supérieur à 90 jours (après le vêlage) on pourrait se poser la question y a-t-il un problème de mise à la reproduction (mauvaise détection de chaleurs, déficit énergétique ou problème sanitaire). En fin de compte le pourquoi du comment reste facile à expliquer l'éleveur veut mettre ses animaux à la reproduction lorsqu'ils se sont "refaîtes" pour avoir plus de chance de réussite.

L'explication donner au-dessus se confirme car le taux de réussite en lère en IA ma paraît très convenable car on a plus de $56 \%$ et plus de $25 \%$ en 2ème IA donc une bonne fertilité du troupeau laitier" (Jordenn).

Le texte reproduit ci-dessus, au-delà des maladresses de l'élève, atteste d'un pluri-référencement prenant sa source dans des indicateurs choisis par l'élève (le nombre d'IA et la période à laquelle elles sont pratiquées), dans des pourcentages pour rendre compte d'une cohérence entre des éléments non habituels (de la mise en évidence d'un écart), dans des propos rapportés du maitre de stage ("En fin de compte le pourquoi du comment reste facile à expliquer l'éleveur veut mettre ses animaux à la reproduction lorsqu'ils se sont "refaîtes" pour avoir plus de chance de réussite"). 


\subsection{De la technique à la science appliquée}

Les élèves du lycée s'appuient sur des processus, des savoirs pour expliciter des pratiques d'élevage liées à ces cycles. Ci-dessus, l'élève traite les objets techniques sur un registre scientifique scolaire (Harlé, 2009). Il met en circulation un ensemble de pratiques au regard de leur opérationnalité (le meilleur rendement possible permis par ces pratiques) dans un schéma énonciatif propre à celui de la science appliquée : décrire des constats, formuler des hypothèses (mauvaise détection de chaleur, déficit énergétique ou problème sanitaire), mettre à l'épreuve ses hypothèses, même si ces dernières ne sont pas toutes traitées. Ce schéma de la science appliquée est d'autant plus légitime qu'il hérite du modèle de la formation des élites agricoles (Grignon, 1975).

Dans l'exemple qui suit, la mise en avant des savoirs de la science appliquée scolaire devient l'articulation par laquelle l'élève répond à la problématique qu'elle pose :

"Le pourcentage d'infertilité a augmenté de $10 \%$ en 2008-2009, du à une chute de $32 \%$ des vaches fécondes en lère IA. L'intervalle vêlage-vêlage a augmenté de 11 jours en 2008-2009. L'année 2008-2009 est plutôt négative, au niveau des résultats ceux-ci séexplique par les problèmes sanitaires rencontrés sur l'exploitation (...). Pour la réforme, il y a eu une augmentation de $9 \%$ du taux de réforme en 2008 du à des problèmes sanitaires (FCO et paratuberculose) qui ont touchés le troupeau laitier. " (Émilie)

Bien que le questionnement n'apparaisse pas de façon aussi explicite que dans la production précédente, cette élève tente de trouver des raisons aux variations constatées. Cette centration sur les savoirs de la science appliquée scolaire est investie encore par cet autre élève :

"La part la plus importante des réformes est l'infertilité. Cela peut ce comprendre a un niveau d'étable de $9500 \mathrm{~kg}$ de lait. De plus, les boiteries on a une part importante dans le taux de réforme car il y a une présence de dermatite sur l'exploitation. " (Arnauld)

La mise en correspondance entre savoirs scientifiques (sanitaires : dermatite, paratuberculose, poids de l'animal comme cause d'infertilité), techniques et pratiques compose les raisonnements les plus fréquents au lycée. Participe encore de cette analyse, la hiérarchisation des énoncés des élèves construits sur la description, permettant de mettre en ouvre un questionnement, de poser des hypothèses et de les tester avec des savoirs techniques ou scientifiques étayés par des éléments objectifs (statistiques, graphiques, etc.). La pluralité des éléments pris en compte (statistiques, propos rapportés, inférences, hypothèses, savoirs mobilisés, mais aussi l'intégration de schémas et graphiques construits) par les élèves tend à dessiner des écrits scientifiques scolaires en prise avec les mutations contemporaines du métier.

Les extraits précédents de Ewann et de Valentyn montrent, à l'opposé, que le système de référence se limite au couple espace/temps empiriquement vécu : l'espace est celui du face-à-face et du voisinage ("la maison d'à côté, le pré, en restant avec les animaux »...), le temps est linéaire ou cyclique ("tous les soirs, vers $22 \mathrm{~h}$, au bout de 
50 jours »...). Enfin, concernant la pluralité des références, elle n'est pas totalement absente mais fait l'objet d'une personnalisation : l'inséminateur et le vétérinaire à qui on peut s'en remettre pour un contrôle chez Ewann, le contrôleur laitier et l'éleveur pour Valentyn.

Ces quelques éléments de l'analyse questionnent l'alternance qui semble avoir des effets différenciés sur les productions des élèves, comme le révèlent les apprentissages tels qu'ils s'actualisent dans leurs écrits. À mi-parcours, les registres mobilisés sont à dominante narrative, de manière linéaire ou cyclique, pour les élèves de MFR, alors qu'ils sont à dominante explicative, sur un registre technique puis déductif, pour les élèves du lycée.

\section{Conclusion : littératies de l'alternance et des institutions}

L'analyse des écrits scolaires en termes de littératie, c'est-à-dire comme pratiques à la fois cognitives et langagières socialement situées, est éclairante au moins à trois titres. Tout d'abord, les écrits peuvent être considérés comme une trace de reprise éventuelle de l'expérience de travail agricole et scolaire, contribuant à structurer une pensée plus ou moins distante de cette expérience. Ensuite, les variations constatées d'un public à l'autre attestent de la coexistence d'une pluralité de littératies scolaires propres à la diversité des dispositifs d'alternance de l'enseignement agricole : l'une à dominante académique, axée sur une rationalité par les savoirs ; la seconde, à dominante expérientielle, axée sur la sociabilité de métier (Malglaive, Weber, 1982 ; 1983). Ces différences ont des effets cognitifs institutionnalisés, dès lors qu'elles sont en concordance avec une origine sociale différenciée des élèves et qu'elles destinent plutôt à des rationalités de types techniciste d'un côté, de communautés de métier, de l'autre.

Enfin, dans les deux études de cas, les types de littératie mises en ouvre sont paradoxalement favorisés par la tendance à la référentialisation des diplômes et à leur mode d'évaluation officiellement autorisé, au moins sous l'angle de l'importance accordée à l'écrit dans la part de la notation finale. Dans cette perspective, la présence de "Contrôles Certificatifs en cours de Formation " (CCF), modulables en fonction des capacités supposées des élèves, favorise les écarts sous couvert d'un diplôme unique.

Ce type de prescription curriculaire encourage sans doute des ajustements localisés, des normes implicites de diplôme, des tolérances «bienveillantes " à l'égard des écrits des élèves. Soulignons d'ailleurs que les écrits scolaires ont comme spécificité d'être un vecteur de co-production par le biais de corrections, de demandes de développements formulées au moins par l'enseignant. À cet égard, il serait pertinent d'interroger le rôle des enseignants de la MFR et du lycée, puisque les productions écrites diffèrent fortement d'un groupe d'élèves à l'autre et qu'en outre, les écarts s'accroissent de façon longitudinale. 
En effet, les travaux de l'équipe EScol (Education et Scolarisation) suggèrent que les bienveillances sont davantage le fait d'un enseignement orienté vers les jeunes de milieux populaires, ouvriers et employés ; le sont-elles plus encore lorsque ces mêmes jeunes sont relégués en lisière du système éducatif et de ses nouveaux standards de diplôme, suite à la réforme du bac pro et plus largement de l'avènement de "la société des diplômes" (Millet, Moreau, 2011) ?

\section{Bibliographie}

Agulhon C. (2000), "L'alternance : une notion polymorphe, des enjeux et des pratiques segmentés ", Revue française de pédagogie, Vol. 131, Les formations professionnelles entre l'Ecole et l'Entreprise, pp. 55-63.

Barton D., Hamilton M. (2010), "La littératie : une pratique sociale », Langage et Société, 133, pp. 45-62.

Bautier, É. (2009), "Quand le discours pédagogique entrave la construction des usages littéraciés du langage ", Pratiques, 143-144, pp. 11-26.

Bernstein B. (1975), Langage et classes sociales. Codes socio-linguistiques et contrôle social, Paris, Minuit.

Bonnery S. (2007), Comprendre l'échec scolaire, élèves en difficultés et dispositifs pédagogiques, Paris, La Dispute, coll. "L'enjeu scolaire ».

Bourdieu P. (1996), Raisons pratiques, Paris, Seuil, coll. « Points ».

Boutet, J. (2008), La vie verbale au travail, des manufactures aux centres d'appels, Toulouse, Octares.

Budach G., Patrick D. (2012), "Chaque objet raconte une histoire ; les pratiques de littératie chez des Inuits en milieu urbain ", Recherches en didactique des langues et des cultures: Les Cahiers de l'Acedle, vol. 9, $\mathrm{n}^{\circ} 2$.

Charlot B., Figeat M. (1985), Histoire de la formation des ouvriers, Paris, Minerve.

Chartier D. (1986), À l'aube des formations par alternance, Paris, Ed. Universitaires, coll. "Mésonance ".

Déotte J.-L. (2010), « Bourdieu et Panofsky : l'appareil de l'habitus scolastique », Appareil [En ligne].

Direction générale de l'Enseignement et de la Recherche, (2013), Panorama de l'Enseignement Agricole.

Forquin J.-C. (2008), Sociologie du curriculum, Rennes, Presses Universitaires de Rennes.

Goigoux R. (2000), Les élèves en grande difficulté de lecture et les enseignements adaptés, Rapport de recherche, ministère de l'Éducation nationale, Éditions du CNEFEI. 
Goody J. (2007), Savoirs et pouvoirs de l'écrit, Paris, La Dispute.

Grignon C. (1975), "L'enseignement agricole et la domination symbolique de la paysannerie ", Actes de la Recherche en Sciences Sociales, n ${ }^{\circ} 1$, Hiérarchie sociale des objets., pp. 75-97.

Harlé I. (2009), La fabrique des savoirs scolaires, La Dispute, coll. « L'enjeu scolaire ».

Heath S.B. (1986), Issues of parent involvement and literacy. Communication présentée dans le cadre du Symposium de Trinity College, Trinity College, Washington, DC, 6-7 juin.

Lahire B. (1993), Culture écrite et inégalités scolaires, Lyon, Presses Universitaires de Lyon.

Lelorrain A.-M. (1999), "L'enseignement postscolaire agricole ", in L'enseignement agricole : 150 ans d'histoire, évolution historique et atlas contemporain, Charmasson T., Duvigneau M., Lelorrain A.-M., Le Naou H. (eds), Dijon, Educagri éditions.

Malglaive G. , Weber A. (1983), "École et entreprise [intérêt et limites de l'alternance en pédagogie - l'alternance emploi-formation ", Revue française de pédagogie, Vol. 62, pp. 51-64.

Malglaive G., Weber A. (1982), "Théorie et pratique, approche critique de l'alternance en pédagogie ", Revue française de pédagogie, Vol. 61, pp. 17-27.

Mangez É. (2008), Réformer les contenus d'enseignement, Paris , PUF.

Millet M., Moreau G. (2001), La société des diplômes, Millet M. \& Moreau G. (dir.), Paris, La Dispute.

Minassian L. (2015), " Permanence des ordres d'enseignement : réseaux, orientation et classes sociales. L'exemple de la formation agricole ", Recherches en Éducation, H-S 7, pp. 111-124.

Merhan F., Ronveaux C., Vanhulle S. (dir.) (2007), «Alternances en formation », Raisons Educatives, Bruxelles, De Boeck Université.

Normand A. (2014), "Proposition pour l'induction en analyse du discours ", Approches inductives: Travail intellectuel et construction des connaissances, $\mathrm{n}^{\circ} 1$, pp. 11-37.

Olson D.R. (1977), "From utterance to text : The bias of language in speech and writing", Harvard Educational Review, 47(3), pp. 257-81.

Panosky E. (1967), Architecture gothique et pensée scolastique, (préface). L'Abbé Suger de Saint-Denis, trad. et postfacé par Pierre Bourdieu, Paris, Éditions de Minuit.

Schribner S., Cole M. (1981), The Psychology of literacy, Cambridg, Havard University Press.

Street B. (2003), "What's new in the New Literacy Studies? Critical approaches to literacy in theory and practice", Current Issues in Comparative Education, 5(2), pp. 77-91. 
Street B. (1995), Social literacies: Critical approaches to literacy in development, ethnography, and education, Londres, Longman.

Street B. (1993), Cross-cultural Approaches to Literacy, Cambridge, Cambridge University Press.

Street B. (1984), Literacy in theory and practice. Cambridge, Cambridge University Press.

Zaffran J. (2010), « Entrer en SEGPA et en sortir ou la question des inégalités transposées ", Formation Emploi n ${ }^{\circ} 109$, La Documentation française, pp. 85-97. 